\title{
Higher Algebraic $K$-theory for Twisted Laurent Series Rings Over Orders and Semisimple Algebras
}

\section{Aderemi Kuku}

Received: 5 May 2007 / Accepted: 9 July 2007 / Published online: 28 May 2008

(C) Springer Science + Business Media B.V. 2008

\begin{abstract}
Let $R$ be the ring of integers in a number field $F, \Lambda$ any $R$-order in a semisimple $F$-algebra $\Sigma, \alpha$ an $R$-automorphism of $\Lambda$. Denote the extension of $\alpha$ to $\Sigma$ also by $\alpha$. Let $\Lambda_{\alpha}[T]$ (resp. $\Sigma_{\alpha}[T]$ be the $\alpha$-twisted Laurent series ring over $\Lambda$ (resp. $\Sigma$ ). In this paper we prove that (i) There exist isomorphisms $\mathbb{Q} \otimes K_{n}\left(\Lambda_{\alpha}[T]\right) \simeq \mathbb{Q} \otimes$ $G_{n}\left(\Lambda_{\alpha}[T]\right) \simeq \mathbb{Q} \otimes K_{n}\left(\Sigma_{\alpha}[T]\right)$ for all $n \geq 1$. (ii) $G_{n}^{\mathrm{pr}}\left(\Lambda_{\alpha}[T], \hat{Z}_{l}\right) \simeq G_{n}\left(\Lambda_{\alpha}[T], \hat{Z}_{l}\right)$ is an $l$-complete profinite Abelian group for all $n \geq 2$. (iii) $\operatorname{div} G_{n}^{\mathrm{pr}}\left(\Lambda_{\alpha}[T], \hat{Z}_{l}\right)=0$ for all $n \geq 2$. (iv) $G_{n}\left(\Lambda_{\alpha}[T]\right) \longrightarrow G_{n}^{\mathrm{pr}}\left(\Lambda_{\alpha}[T], \hat{Z}_{l}\right)$ is injective with uniquely $l$-divisible cokernel (for all $n \geq 2$ ). (v) $K_{-1}(\Lambda), K_{-1}\left(\Lambda_{\alpha}[T]\right.$ ) are finitely generated Abelian groups.
\end{abstract}

Keywords $K$-theory $\cdot$ Twisted Laurent series rings $\cdot$ Semisimple algebras • Orders • Virtually infinite cyclic group

Mathematics Subject Classifications (2000) $19 \mathrm{D} 35 \cdot 16 \mathrm{~S} 35 \cdot 16 \mathrm{H} 05 \cdot 16 \mathrm{~S} 34$

\section{Introduction}

Let $R$ be the ring of integers in a number field $F$. The initial motivation for this work was a desire to obtain results on higher $K$-theory of the groupring $R V$ of a virtually infinite cyclic group of the form $V=G \rtimes_{\alpha} T$, where $G$ is a finite group, $\alpha$

Presented by Alain Verschoren.

A. Kuku $(\varangle)$

Mathematics Department, The University of Iowa,

14 Maclane Hall, Iowa City, Iowa 52242, USA

e-mail: akuku@math.uiowa.edu

A. Kuku

Max-Planck-Institut für Mathematik, Bonn, Germany 
an automorphism of $G$ and the action of the infinite cyclic group $T=\langle t\rangle$ on $G$ is given by $\alpha(g)=\operatorname{tgt}^{-1}$ for all $g \in G$.

Note that understanding the $K$-theory of $R V$ is fundamental to the Farrell-Jones conjecture which asserts that $K$-theory of an arbitrary discrete group $H$ should have as "building blocks" the $K$-theory of virtually cyclic subgroups of $H$ (see [8]). A group $V$ is virtually cyclic if it is either finite or virtually infinite cyclic (i.e., contains a finite index subgroup that is infinite cyclic). For results on higher $K$-theory of grouprings of finite groups see [15, chapter 7] and associated references. There are two types of virtually infinite cyclic groups - one type of the form $V=G \rtimes_{\alpha} T$ as described above and the other of the form $V=G_{0} *_{H} G_{1}$, where the groups $G_{0}, G_{1}$, $H$ are finite and $\left[G_{0}: H\right]=\left[G_{1}: H\right]=2$. For some results on higher $K$-theory of both types of groups see [15, 7.5] or [16]. In this paper, we obtain results on higher $K$-theory of twisted Laurent series ring that translate into results on grouprings $R V$, $V=G \rtimes_{\alpha} T$, as we now explain.

If $\alpha$ is an automorphism of a finite group $G$, we also denote by $\alpha$ the automorphism induced on $R G$ by $\alpha$ and observe that for $V=G_{\alpha} \rtimes T, R V=(R G)_{\alpha}[T]=$ $(R G)_{\alpha}\left[t, t^{-1}\right]$ is the $\alpha$-twisted Laurent series ring over the groupring $R G$. Now, $R G$ is an $R$-order in the semi-simple $F$-algebra $F G$ and so, we endeavour in this paper to obtain general results on higher $K$-theory of $\Lambda_{\alpha}(T)$ where $\Lambda$ is an arbitrary $R$-order in a semi-simple $F$-algebra $\Sigma$ so that results on $(R G)_{\alpha}[T]$ become examples and applications of our results.

Note also that an $R$-automorphism of $\Lambda$ extends to an $F$-automorphism of $\Sigma$ which we also denote by $\alpha$. We also study higher $K$-theory of $\Sigma_{\alpha}[T]$ and prove in Theorem 1(b) that there exist isomorphisms

$$
\mathbb{Q} \otimes K_{n}\left(\Lambda_{\alpha}[T]\right) \simeq \mathbb{Q} \otimes G_{n}\left(\Lambda_{\alpha}[T]\right) \simeq \mathbb{Q} \otimes K_{n}\left(\Sigma_{\alpha}[T]\right)
$$

for all $n \geq 2$. Hence $\mathbb{Q} \otimes K_{n}(R V) \simeq \mathbb{Q} \otimes G_{n}(R V) \simeq Q \otimes K_{n}(F V)$ for all $n \geq 2$. Since we have shown in Theorem 1 (a) that $G_{n}\left(\Lambda_{\alpha}[T]\right)$ is a finitely generated Abelian group for all $n \geq 1$, it follows that $K_{n}\left(\Lambda_{\alpha}[T]\right), K_{n}\left(\Sigma_{\alpha}[T]\right)$ and hence $K_{n}(R V), K_{n}(F V)$ have finite torsion-free ranks for all $n \geq 2$.

We next investigate under what conditions $G_{n}\left(\Lambda_{\alpha}[T]\right)$ could actually be a finite group and show in Theorem 6 that when $F$ is a totally real number field with ring of integers $R$ and $\Lambda$ any $R$-order in a semi-simple $F$-algebra, then $G_{2(m+1)}\left(\Lambda_{\alpha}[T]\right)$ is finite for all odd $m \geq 1$. Hence $G_{2(m+1)}(R V)$ is finite.

In Section 3, we study profinite higher $K$-theory of $\Lambda_{\alpha}[T]$ and prove that $G_{n}^{\mathrm{pr}}\left(\Lambda_{\alpha}[T], \hat{\mathbb{Z}}_{l}\right)=G_{n}\left(\Lambda_{\alpha}[T], \hat{\mathbb{Z}}_{l}\right)$ are $l$-complete profinite Abelian groups; $\operatorname{div} G_{n}^{\mathrm{pr}}\left(\Lambda_{\alpha}[T], \hat{\mathbb{Z}}_{l}\right)=0$; and that the map $G_{n}\left(\Lambda_{\alpha}[T]\right) \longrightarrow G_{n}^{\mathrm{pr}}\left(\Lambda_{\alpha}[T], \hat{\mathbb{Z}}_{l}\right)$ is injective with uniquely $l$-divisible cokernel. Corresponding results follow when we replace $\Lambda_{\alpha}[T]$ by $R V$.

In a final section, we prove that if $F$ is an algebraic number field with ring of integers $R$ and $\Lambda$ any $R$-order in a semi-simple $F$-algebra $\Sigma$, then $K_{-1}(\Lambda)$ and $K_{-1}\left(\Lambda_{\alpha}[T]\right)$ are finitely generated Abelian groups; $N K_{-1}(\Lambda, \alpha)=0$ and $K_{-1}(\Lambda[t]) \simeq$ $K_{-1}(\Lambda)$. That $K_{-1}(\Lambda)$ and $K_{-1}\left(\Lambda_{\alpha}[T]\right)$ are finitely generated for arbitrary $R$-orders $\Lambda$ generalizes similar results by D. Carter for $K_{-1}(R G)$ ( $G$ a finite group, see [4]) resp. by Farrell/Jones for $K_{-1}(\mathbb{Z} V)$ (see [9]).

Notes on notation If $\alpha$ is an automorphism of a ring $A$, we shall write $A_{\alpha}[T]=$ $A_{\alpha}\left[t, t^{-1}\right]$ for the $\alpha$-twisted Laurent series ring over $A$. Note that additively $A_{\alpha}[T]=$ 空 Springer 
$A_{\alpha}\left[t, t^{-1}\right]$ with multiplication given by $\left(a t^{i}\right) \cdot\left(b t^{j}\right)=a \alpha^{-1}(b) t^{i+j}$ for $a, b \in A . A_{\alpha}[t]$ (resp. $A_{\alpha}\left[t^{-1}\right]$ ) is the subring of $A_{\alpha}[T]$ generated by $A$ and $t$ (resp. $A$ and $t^{-1}$ ). Call $A_{\alpha}[t]$ the $\alpha$-twisted polynomial ring over $A$. We also have inclusion maps $i: A \rightarrow$ $A_{\alpha}[T], i^{+}: A \rightarrow A_{\alpha}[t]$ and $i^{-}: A \rightarrow A_{\alpha}\left[t^{-1}\right]$.

The augmentation map $\varepsilon: A_{\alpha}[t] \rightarrow A$ induces a group homomorphism $\varepsilon_{*}$ : $K_{n}\left(A_{\alpha}[t]\right) \rightarrow K_{n}(A)$ and we put $N K_{n}(A, \alpha):=\operatorname{ker} \varepsilon_{*}$. Since $\varepsilon$ is split by $i^{+}$, we have $K_{n}\left(A_{\alpha}[t]\right) \simeq K_{n}(A) \oplus N K_{n}(A, \alpha)$.

If $B$ is an additive Abelian group and $m$ is a positive integer, we shall write $B / m$ for $B / m B$ and $B[m]$ for the set of elements $x$ of $B$ such that $m x=0$. We write div $B$ for the subgroup of divisible elements of $B$. If $l$ is a rational prime, we write $B_{l}$ for the $l$-primary subgroup of $B$. Note that $B_{l}=\bigcup B\left[l^{s}\right]=\underset{\lim }{\longrightarrow} B\left[l^{s}\right]$.

\section{Higher $K$-theory of $\Lambda_{\alpha}[T], \Sigma_{\alpha}[T]$ ( $\Lambda$ Arbitrary Orders)}

\section{1. $K_{n}\left(\Lambda_{\alpha}[T]\right), G_{n}\left(\Lambda_{\alpha}[T]\right), K_{n}\left(\Sigma_{\alpha}[T]\right)$}

2.1.1 Let $R$ be the ring of integers in a number field $F, \Lambda$ any $R$-order in a semisimple $F$-algebra $\Sigma, \alpha$ an $R$-automorphism of $\Lambda$. Then $\alpha$ can be extended to an $F$-automorphism of $\Sigma$ (since $\Sigma=\Lambda \otimes_{R} F$ ). The aim of this section is to prove the following theorem.

Theorem 1 Let $F$ be an algebraic number field with ring of integers $R, \Lambda$ any $R$-order in a semi-aimple F-algebra $\Sigma, \alpha$ an $R$-automorphism of $\Lambda$. Denote the extension of $\alpha$ to $\Sigma$ also by $\alpha$. Let $\Lambda_{\alpha}[T]$ (resp. $\left.\Sigma_{\alpha}[T]\right)$ be the $\alpha$-twisted Laurent series ring over $\Lambda$ (resp. $\Sigma$ ). Then we have

(a) $G_{n}\left(\Lambda_{\alpha}[T]\right)$ is a finitely generated Abelian group for all $n \geq 1$.

(b) There exist isomorphisms:

$$
\mathbb{Q} \otimes K_{n}\left(\Lambda_{\alpha}[T]\right) \simeq \mathbb{Q} \otimes G_{n}\left(\Lambda_{\alpha}[T]\right) \simeq \mathbb{Q} \otimes K_{n}\left(\Sigma_{\alpha}[T]\right)
$$

for $n \geq 2$.

Before proving Theorem 1 we state the following consequence of the result.

Corollary 1 Let $V=G \rtimes_{\alpha} T$ be the virtually infinite cyclic subgroup where $G$ is a finite group, $\alpha \in \operatorname{Aut}(G)$ and the action of $T$ on $G$ is given by $\alpha(g)=\operatorname{tgt}^{-1}$, for all $g \in G$. Then,

(a) $G_{n}(R V)$ is a finitely generated Abelian group for al $n \geq 1$.

(b) $\mathbb{Q} \otimes K_{n}(R V) \simeq \mathbb{Q} \otimes G_{n}(R V) \simeq \mathbb{Q} \otimes K_{n}(F V)$ for all $n \geq 2$.

The proof of Theorem 1(b) will proceed in several steps (see Theorems 3, 4, 5 below). However, we first recall the following result: Theorem 2.

Theorem 2 ([15, Theorem 7.3.2] or [16]) Let $R$ be the ring of integers in a number field $F, \Lambda$ any $R$-order in a semi-simple F-algebra $\Sigma$. If $\alpha: \Lambda \rightarrow \Lambda$ is an R-automorphism, then there exists an $R$-order $\Gamma \subset \Sigma$, such that

(1) $\Lambda \subset \Gamma$;

(2) $\Gamma$ is $\alpha$-invariant;

(3) $\Gamma$ is (right) regular ring. In fact $\Gamma$ is (right) hereditary. 
Theorem 3 Let $R$ be the ring of integers in a number field $F, \Lambda$ any $R$-order in a semi-simple F-algebra, $\alpha: \Lambda \rightarrow \Lambda$ and $R$-automorphism of $\Lambda, \Gamma$ an $\alpha$-invariant order containing $\Lambda$ as in Theorem $2, \Lambda_{\alpha}[T]$ (resp. $\Gamma_{\alpha}[T]$ ) the $\alpha$-twisted Laurent series ring over $\Lambda$ (resp. $\Gamma) . \varphi: \Lambda_{\alpha}[T] \rightarrow \Gamma_{\alpha}[T]$ the map induced by the inclusion $\Lambda \rightarrow \Gamma$. Then the induced homomorphisms $\varphi_{n}: K_{n}\left(\Lambda_{\alpha}[T]\right) \rightarrow K_{n}\left(\Gamma_{\alpha}[T]\right)$ has torsion kernel and cokernel. Hence for all $n \geq 2$ we have $\mathbb{Q} \otimes K_{n}\left(\Lambda_{\alpha}[T]\right) \simeq \mathbb{Q} \otimes K_{n}\left(\Gamma_{\alpha}[T]\right)$.

Proof There exists a positive integer such that $s \Gamma \subset \Lambda$ (see [18] or [15]). Put $q=s \Gamma$. Then $\underline{q}$ is an ideal of $\Gamma$ and $\Lambda$. Put $B=\Lambda / \underline{q}, B^{\prime}=\Gamma / \underline{q}$. Then we have cartesian squares

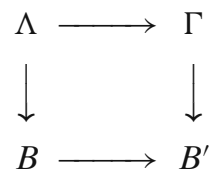

and

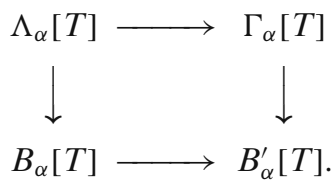

So, by [5] and [19], we have a long exact sequence

$$
\begin{aligned}
\cdots \rightarrow K_{n+1}\left(B_{\alpha}^{\prime}[T]\right)\left(\frac{1}{s}\right) & \longrightarrow K_{n}\left(\Lambda_{\alpha}[T]\right)\left(\frac{1}{s}\right) \\
& \longrightarrow K_{n}\left(\Gamma_{\alpha}[T]\right)\left(\frac{1}{s}\right) \oplus K_{n}\left(B_{\alpha}[T]\right)\left(\frac{1}{s}\right) \\
& \longrightarrow K_{n}\left(B_{\alpha}^{\prime}[T]\right)\left(\frac{1}{s}\right) \rightarrow \cdots
\end{aligned}
$$

Now, $\Gamma, \mathrm{B}, \mathrm{B}^{\prime}$ are quasi-regular rings, so are $\Gamma_{\alpha}[T], B_{\alpha}[T]$ and $B_{\alpha}^{\prime}[T]$ (see [9]). If we write $A$ for $B_{\alpha}[T]$ or $B_{\alpha}^{\prime}[T], J A$ for the Jacobson's radical of $A$, then by [19] $K_{n}(A, J A)$ is $s$-torsion since $s$ annihilates $A$ and so from the relative sequence

$$
\cdots \longrightarrow K_{n}(A, J A) \longrightarrow K_{n}(A) \longrightarrow K_{n}(A / J) \longrightarrow \cdots
$$

we have $K_{n}(A)\left(\frac{1}{s}\right) \simeq K_{n}(A / J A)\left(\frac{1}{s}\right)$. We now claim that $K_{n}(A)\left(\frac{1}{s}\right) \simeq K_{n}(A / J A)\left(\frac{1}{s}\right)$ is torsion.

Proof of the claim Note that $A / J A \simeq\left(A^{\prime} / J A^{\prime}\right)_{\alpha}[T]$ is a regular ring (see [9]) where $A^{\prime} / J A^{\prime}$ is a finite semi-simple ring which is a finite direct product of matrix algebras over finite fields. Hence $K_{n}\left(\left(A^{\prime} / J A^{\prime}\right)_{\alpha}[T]\right)$ is a finite direct sum of $K$-groups of the form $K_{n}\left(\left(F_{i}\right)_{\alpha}[T]\right)$ where $F_{i}$ is a finite field. Also, $\left(F_{i}\right)_{\alpha}[T]$ is a regular ring and so $K_{n}\left(\left(F_{i}\right)_{\alpha}[T]\right) \simeq G_{n}\left(\left(F_{i}\right)_{\alpha}[T]\right)$. 
Now, for each $F_{i}$, we have by [15, Theorem 7.5.3(iii)] or [16], that there exists a long exact sequence

$$
\begin{aligned}
\cdots \rightarrow G_{n}\left(F_{i}\right) & \longrightarrow G_{n}\left(F_{i}\right) \longrightarrow G_{n}\left(\left(F_{i}\right)_{\alpha}[T]\right) \\
& \longrightarrow G_{n-1}\left(F_{i}\right) \longrightarrow G_{n-1}\left(F_{i}\right) \rightarrow \cdots
\end{aligned}
$$

where each $G_{n}\left(F_{i}\right) \simeq K_{n}\left(F_{i}\right)$ is a finite Abelian group for $n \geq 2-$ by [15, Theorem 7.1.12] or by Quillen's result. So, from Eq. 4 above, $G_{n}\left(\left(F_{i}\right)_{\alpha}[T]\right)$ is finite for all $n \geq 2$, i.e. $K_{n}\left(\left(F_{i}\right)_{\alpha}[T]\right) \simeq G_{n}\left(\left(F_{i}\right)_{\alpha}[T]\right)$ is a finite Abelian group. Hence $\left(K_{n}\left(A^{\prime} / J A^{\prime}\right)_{\alpha}[T]\right)$, as a finite direct sum of Abelian groups of the form $K_{n}\left(F_{i}\right)_{\alpha}[T]$ is a finite group. Hence $K_{n}\left(\left(A^{\prime} / J A^{\prime}\right)_{\alpha}[T]\right)\left(\frac{1}{s}\right)$ is torsion. So, for $A=B_{\alpha}(T)$ or $B_{\alpha}^{\prime}[T], K_{n}(A)\left(\frac{1}{s}\right) \simeq$ $K_{n}\left((A / J A)\left(\frac{1}{s}\right)\right)$ is torsion and $\mathbb{Q} \otimes K_{n}(A)\left(\frac{1}{s}\right)=0$.

So, by tensoring the Mayer-Vietoris exact sequence Eq. 3 with $\mathbb{Q}$ we get an isomorphism

$$
\mathbb{Q} \otimes K_{n}\left(\Lambda_{\alpha}[T]\right) \simeq \mathbb{Q} \otimes K_{n}\left(\Gamma_{\alpha}[T]\right)
$$

for all $n \geq 2$.

Theorem 4 Let $R, F, \Lambda, \alpha ; \Gamma, \Lambda_{\alpha}[T], \Gamma_{\alpha}[T]$ be as in Theorem 3. Let $\varphi_{n}$ : $G_{n}\left(\Gamma_{\alpha}[T]\right) \longrightarrow G_{n}\left(\Lambda_{\alpha}[T]\right)$ be the homomorphism induced by the exact functor $\mathcal{M}\left(\Gamma_{\alpha}[T]\right) \rightarrow \mathcal{M}\left(\Lambda_{\alpha}[T]\right)$ given by 'restriction of scalars'. Then for all $n \geq 2, \varphi_{n}$ has finite kernel and torsion cokernel and hence induces an isomorphism

$$
\mathbb{Q} \otimes G_{n}\left(\Gamma_{\alpha}[T]\right) \simeq \mathbb{Q} \otimes G_{n}\left(\Lambda_{\alpha}[T]\right)
$$

Proof First note that the exact functor $\mathcal{M}(\Gamma) \rightarrow \mathcal{M}(\Lambda)$ given by 'restriction of scalars' yields group homomorphisms $\delta_{n}: G_{n}(\Gamma) \rightarrow G_{n}(\Lambda)$. Now, by replacing the maximal order $\Gamma$ in the proof of [15, Theorem 7.2.3, p. 146] or [16] with the $\alpha$ invariant order $\Gamma$ containing $\Lambda$, as in Theorem 2 , we have that for all $n \geq 1, \delta_{n}$ : $G_{n}(\Gamma) \rightarrow G_{n}(\Lambda)$ has finite kernel and cokernel. The proof in [15, Theorem 7.2.3] works for this $\Gamma$ also. Now from [15, Theorem 7.5.3(b)] or [16], we have the following horizontal exact sequence and hence a commutative diagram

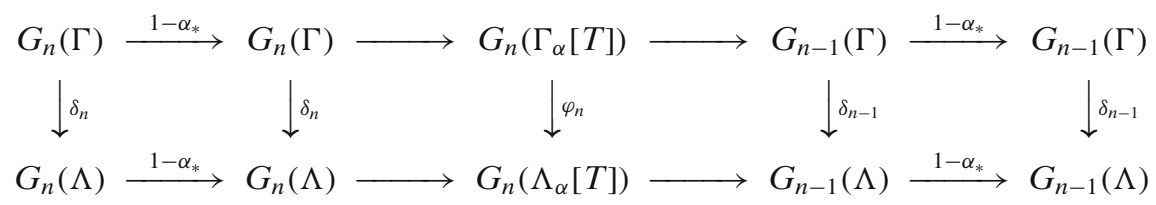

By taking kernels and cokernels of vertical arrows in Eq. 5, we have a top (resp. bottom) horizontal exact sequence consisting of kernels (resp. cokernels) of the vertical maps. Since we saw above that $\delta_{n}$ has finite kernels and cokernels, we then have that $\phi_{n}: G_{n}\left(\Gamma_{\alpha}[T]\right) \rightarrow G_{n}\left(\Lambda_{\alpha}[T]\right)$ has finite kernel and cokernel for each $n \geq 2$. Hence $\mathbb{Q} \otimes G_{n}\left(\Gamma_{\alpha}[T]\right) \simeq \mathbb{Q} \otimes G_{n}\left(\Lambda_{\alpha}[T]\right)$. But $\Gamma_{\alpha}[T]$ is regular. Hence

$$
\mathbb{Q} \otimes K_{n}\left(\Gamma_{\alpha}[T]\right) \simeq \mathbb{Q} \otimes G_{n}\left(\Lambda_{\alpha}[T]\right) .
$$


Theorem 5 Let $R, F, \Sigma, \Lambda, \alpha, T$ be as in Theorem 1 . Then for all $n \geq 2$, the map $\theta_{n}$ : $G_{n}\left(\Lambda_{\alpha}[T]\right) \longrightarrow G_{n}\left(\Sigma_{\alpha}[T]\right) \simeq K_{n}\left(\Sigma_{\alpha}[T]\right)$ induced by the canonical map $\Lambda_{\alpha}[T] \rightarrow$ $\Sigma_{\alpha}[T]$ has finite kernel and torsion cokernel. Hence

$$
\mathbb{Q} \otimes G_{n}\left(\Lambda_{\alpha}[T]\right) \simeq \mathbb{Q} \otimes G_{n}\left(\Sigma_{\alpha}[T]\right) \simeq \mathbb{Q} \otimes K_{n}\left(\Sigma_{\alpha}[T]\right)
$$

Proof Note that the canonical (inclusion) map $\Lambda \stackrel{\rho}{\rightarrow} \Sigma$ induces a group homomorphism $\rho_{n}: G_{n}(\Lambda) \rightarrow G_{n}(\Sigma) \simeq K_{n}(\Sigma)$ (note that $G_{n}(\Sigma) \simeq K_{n}(\Sigma)$ since $\Sigma$ is regular).

Now, by [15, Theorem 7.5.3(b)] or [16], we have the following horizontal exact sequences and hence a commutative diagram

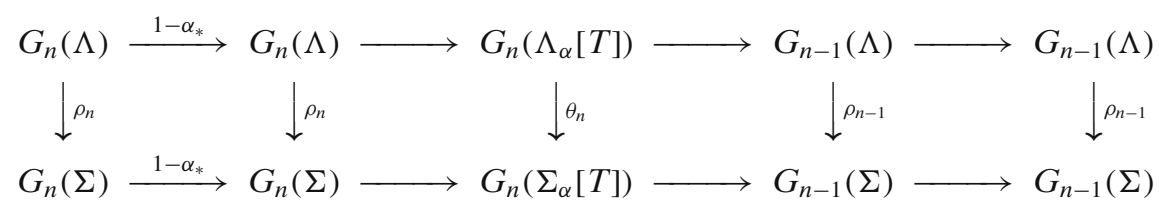

Now, from the commutative diagram

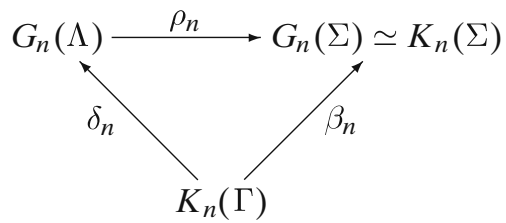

we have

$$
0 \rightarrow \operatorname{ker} \delta_{n} \rightarrow \operatorname{ker} \beta_{n} \rightarrow \operatorname{ker} \rho_{n} \rightarrow \operatorname{coker} \delta_{n} \rightarrow \operatorname{coker} \beta_{n} \rightarrow \operatorname{coker} \rho_{n} \rightarrow 0
$$

Now, by the proof of Theorem $4, \operatorname{ker} \delta_{n}$ and coker $\delta_{n}$ are finite. Also by [15, Theorem 7.2.2] or [12], $\operatorname{ker} \beta_{n}$ is finite and $\operatorname{coker} \beta_{n}$ is torsion for all $n \geq 2$. Hence from diagram Eq. 7 above, $\operatorname{ker} \rho_{n}$ is finite and $\operatorname{coker} \rho_{n}$ is torsion for all $n \geq 2$. It then follows from the diagram Eq. 6 above that $\operatorname{ker} \theta_{n}$ is finite and $\operatorname{coker} \theta_{n}$ is torsion.

Proof of Theorem 1 (a) From [15, Theorem 7.5.3(b)] or [16], we have an exact sequence

$$
G_{n}(\Lambda) \stackrel{1-\alpha_{*}}{\longrightarrow} G_{n}(\Lambda) \longrightarrow G_{n}\left(\Lambda_{\alpha}[T]\right) \longrightarrow G_{n-1}(\Lambda) \stackrel{1-\alpha_{*}}{\longrightarrow} G_{n-1}(\Lambda)
$$

Also by [15, Theorem 7.1.13] or [10] $G_{n}(\Lambda)$ is a finitely generated Abelian group for all $n \geq 1$. Hence $G_{n}\left(\Lambda_{\alpha}[T]\right)$ is finitely generated for all $n \geq 2$. (b) That $\mathbb{Q} \otimes$ $K_{n}\left(\Lambda_{\alpha}[T]\right) \simeq \mathbb{Q} \otimes G_{n}\left(\Lambda_{\alpha}[T]\right)$ follow from Theorem 2 i.e. $\mathbb{Q} \otimes K_{n}\left(\Lambda_{\alpha}[T]\right) \simeq \mathbb{Q} \otimes$ $K_{n}\left(\Gamma_{\alpha}[T]\right)$ and Theorem 3 i.e. $\mathbb{Q} \otimes G_{n}\left(\Lambda_{\alpha}[T]\right) \simeq \mathbb{Q} \otimes K_{n}\left(\Sigma_{\alpha}[T]\right)$.

Remark 1 Since by Theorem $1(\mathrm{a}), G_{n}\left(\Lambda_{\alpha}[T]\right)$ is finitely generated Abelian group for all $n \geq 2$, it follows that $K_{n}\left(\Lambda_{\alpha}[T]\right)$ and $K_{n}\left(\Sigma_{\alpha}[T]\right)$ have finite torsion free rank just like $G_{n}\left(\Lambda_{\alpha}[T]\right)$.

Hence if $V=G \rtimes_{\alpha} T$ is a virtually infinite cyclic group, then $K_{n}(R V), K_{n}(F V)$ have finite torsion-free rank for $n \geq 2$. 


\subsection{Finiteness of $G_{2(m+1)}\left(\Lambda_{\alpha}[T]\right)$}

In this subsection, we investigate under what circumstances $G_{n}\left(\Lambda_{\alpha}[T]\right)$ could actually be a finite group. We prove below (see Theorem 6) that if $F$ is a totally real field, then the group $G_{2(m+1)}\left(\Lambda_{\alpha}[T]\right)$ is finite for all odd positive integers $m$. We state this formally:

Theorem 6 Let $R$ be the ring of integers in a totally real number field $F, \Lambda$ an $R$-order in a semi-simple F-algebra, $\alpha: \Lambda \rightarrow \Lambda$ and $R$-automorsphim. Then for all odd positive integers $m, G_{2(m+1)}\left(\Lambda_{\alpha}[T]\right)$ is a finite group. Hence in the notation of Theorem 1, $G_{2(m+1)}(R V)$ is finite.

The proof of Theorem 6 will make use of the following:

Theorem 7 Let $F$ be a number field with ring of integers $R, \Lambda$ and $R$-order in a semisimple F-algebra $\Sigma$. Then (a) For all $n \geq 1, G_{2 n}(\Lambda)$ is a finite group. (b) If $F$ is totally real, then $G_{2 m+1}(\Lambda)$ is also finite for all odd $m \geq 1$.

Proof Part (a) is proved in [15] and [14]. See [15, Theorem 7.2.7].

If $F$ is a totally real number field with ring of integers $O_{F}$, a similar proof works. We only have to show that $K_{2 m+1}(\Gamma)$ is finite if $\Gamma$ is a maximal order in a central division algebra $D$ over a totally real number field $F$ with ring of integer $O_{F}$. Let the dimension of $D$ over $F$ be $s^{2}$. We know from [15, Theorem 7.1.11] or [11] that $K_{2 m+1}(\Gamma)$ is finitely generated. We only need to show that $K_{2 m+1}(\Gamma)$ is torsion. Let tr $: K_{2 m+1}(\Gamma) \rightarrow K_{2 m+1}\left(O_{F}\right)$ be the transfer map and $i: K_{2 m+1}\left(O_{F}\right) \rightarrow K_{2 m+1}(\Gamma)$ the map induced by the inclusion map $O_{F} \rightarrow \Gamma$. Let $x \in K_{2 m+1}(\Gamma)$. Then $i \circ \operatorname{tr}(x)=x^{s^{2}}$. But $K_{2 m+1}(\Gamma)$ is finite since it is also finitely generated. (See [2] for the proof that $K_{2 m+1}\left(O_{F}\right)$ is torsion).

Proof of Theorem 6 Assume that $m$ is an odd positive integer. The we have an exact sequence

$$
\cdots \rightarrow G_{2 m+2}(\Lambda) \stackrel{1-\alpha_{n}}{\longrightarrow} G_{2 m+2}(\Lambda) \stackrel{\beta}{\rightarrow} G_{2 m+2}\left(\Lambda_{\alpha}[T]\right) \stackrel{\gamma}{\rightarrow} G_{2 m+1}(\Lambda) \rightarrow \cdots
$$

where $G_{2 m+2}(\Lambda)$ is finite by Theorem 7(a) and $G_{2 m+1}(\Lambda)$ is finite by Theorem 7(b). So $G_{2 m+2}\left(\Lambda_{\alpha}[T]\right) / \operatorname{Im} \beta \simeq \operatorname{Im} \gamma$.

But $\operatorname{Im} \beta$ is finite and $\operatorname{Im} \gamma$ is also finite as a subgroup of the finite group $G_{2 m+1}(\Lambda)$. Note that $\operatorname{Im} \beta$ is finite as a homomorphic image of the finite group $G_{2 m+2}(\Lambda)$. Hence $G_{2 m+2}\left(\Lambda_{\alpha}[T]\right)$ is finite for all odd positive integers $m$.

\section{Mod- $l^{s}$ and Profinite Higher $K$-theory of $\Lambda_{\alpha}(T)$}

\subsection{Mod- $l^{s}$ Theory}

3.1.1 Let $\mathcal{C}$ be an exact category, $l$ a rational prime, $s$ a positive integer, $M_{l^{s}}^{n+1}$ the $(n+1)$-dimensional mod- $l^{s}$-space, i.e. the space obtained from $S^{n}$ by attaching an $(n+1)$-cell via a map of degree $l^{s}($ see $[3,15,17])$. 
If $X$ is an $H$-space, let $\left[M_{l^{s}}^{n+1}, X\right]$ be the set of homotopy classes of maps from $M_{l^{s}}^{n+1}$ to $X$.We shall write $\pi_{n+1}\left(X, \mathbb{Z} / l^{s}\right)$ for $\left[M_{l^{s}}^{n+1}, X\right]$. If $\mathcal{C}$ is an exact category and we put $X=B Q \mathcal{C}$, we write $K_{n}\left(\mathcal{C}, \mathbb{Z} / l^{s}\right)$ for $\pi_{n+1}(B Q \mathcal{C})$, we write $K_{n}\left(\mathcal{C}, \mathbb{Z} / l^{s}\right)$ for $\pi_{n+1}\left(\mathcal{C}, \mathbb{Z} / l^{s}\right)$ and $K_{0} \mathcal{C}, \mathbb{Z} / l^{s}$ for $K_{0}(\mathcal{C}) \otimes \mathbb{Z} / l^{s}$. We shall refer to $K_{n}\left(\mathcal{C}, \mathbb{Z} / l^{s}\right)$ as mod- $l^{s}$ $K$-theory of $\mathcal{C}$.

3.1.2 From $[15,8.1 .2]$ or [13], we have an exact sequence

$$
K_{n}(\mathcal{C}) \stackrel{l^{s}}{\rightarrow} K_{n}(\mathcal{C}) \stackrel{\rho}{\longrightarrow} K_{n}\left(\mathcal{C}, \mathbb{Z} / l^{s}\right) \stackrel{\beta}{\longrightarrow} K_{n-1}(\mathcal{C}) \longrightarrow K_{n-1}(\mathcal{C})
$$

and hence a short exact sequence for all $n \geq 2$

$$
0 \longrightarrow K_{n}(\mathcal{C}) / l^{s} \longrightarrow K_{n}\left(\mathcal{C}, \mathbb{Z} / l^{s}\right) \longrightarrow K_{n}(\mathcal{C})\left[l^{s}\right] \longrightarrow 0
$$

where $K_{n}(\mathcal{C})\left[l^{s}\right]=\left\{x \in K_{n}(\mathcal{C}) \mid l^{s} x=0\right\}$.

\section{Example 1}

(i) Let $A$ be a ring with identity and $\mathcal{P}(A)$ the category of finitely generated projective $A$-modules. We write $K_{n}\left(A, \mathbb{Z} / l^{s}\right)$ for $K_{n}\left(\mathcal{P}(A), \mathbb{Z} / l^{S}\right)$. We are interested in $A=\Lambda_{\alpha}(T)$. Note that $K_{n}\left(A, \mathbb{Z} / l^{S}\right)$ is also $\pi_{n}\left(B G L(A)^{+}, \mathbb{Z} / l^{S}\right)$.

(ii) Let $A$ be a Noetherian ring and $\mathcal{M}(A)$ the category of finitely generated $A$ modules. We write $G_{n}\left(A, \mathbb{Z} / l^{s}\right)$ for $K_{n}\left(\mathcal{A}, \mathbb{Z} / l^{s}\right)$.

(iii) Let $Y$ be a scheme, $\mathcal{C}=\mathcal{P}(Y)$ the category of locally free sheaves of $O_{X^{-}}$ modules of finite rank. We write $K_{n}\left(X, \mathbb{Z} / l^{s}\right)$ for $K_{n}\left(\mathcal{P}(Y), \mathbb{Z} / l^{s}\right)$ and observe that for $Y=\operatorname{Spec}(A), A$ a commutative ring, we recover $K_{n}\left(A, \mathbb{Z} / l^{S}\right)$ as in (i).

(iv) Let $Y$ be a Noetherian scheme and $\mathcal{M}(Y)$ the category of coherent sheaves of $O_{Y}$-modules. We write $G_{n}\left(Y, \mathbb{Z} / l^{s}\right)$ for $K_{n}\left(\mathcal{M}\left(Y^{\prime}\right), \mathbb{Z} / l^{s}\right)$ and when $Y=$ $\operatorname{Spec}(A)$, where $A$ is commutative, then we recover $G_{n}\left(A, \mathbb{Z} / l^{S}\right)$ as in (ii) above.

(v) It follows from Section 3.1.2 that we have exact sequences

$$
0 \longrightarrow K_{n}\left(\Lambda_{\alpha}[T]\right) / l^{s} \longrightarrow K_{n}\left(\Lambda_{\alpha}[T], \mathbb{Z} / l^{s}\right) \longrightarrow K_{n}\left(\Lambda_{\alpha}[T]\right)\left[l^{s}\right] \longrightarrow 0
$$

and

$$
0 \longrightarrow G_{n}\left(\Lambda_{\alpha}[T]\right) / l^{s} \longrightarrow G_{n}\left(\Lambda_{\alpha}[T], \mathbb{Z} / l^{S}\right) \longrightarrow G_{n}\left(\Lambda_{\alpha}[T]\right)\left[l^{s}\right] \longrightarrow 0
$$

\subsection{Profinite Higher $K$-theory}

3.2.1 Let $\mathcal{C}$ be an exact category, $l$ a rational prime, $s$ a positive integer $M_{l^{\infty}}^{n+1}=$ $\lim _{\leftarrow} M_{l^{s}}^{n+1}$. We define the profinite $K$-theory of $\mathcal{C}$ by $K_{n}^{\mathrm{pr}}\left(\mathcal{C}, \hat{\mathbb{Z}}_{l}\right):=\left[M_{l^{\infty}}^{n+1}, B Q \mathcal{C}\right]$. We write $K_{n}\left(\mathcal{C}, \hat{\mathbb{Z}}_{l}\right)$ for $\lim _{s} K_{n}\left(\mathcal{C}, \mathbb{Z} / l^{s}\right)$.

For more details on these constructions and their properties, see [15, Chapter 8] or [13].

空 Springer 
Example 2

(i) $\quad$ For $\mathcal{C}=\mathcal{P}(A)$ as in Example $1(\mathrm{i})$, we shall write $K_{n}^{\mathrm{pr}}\left(A, \hat{\mathbb{Z}}_{l}\right)$ for $K_{n}^{\mathrm{pr}}\left(\mathcal{P}(A), \hat{\mathbb{Z}}_{l}\right)$ and $K_{n}\left(A, \hat{\mathbb{Z}}_{l}\right)$ for $K_{n}\left(\mathcal{P}(A), \hat{\mathbb{Z}}_{l}\right)$.

(ii) For $\mathcal{C}=\mathcal{M}(A)$ as in Example 1(ii), we shall write $G_{n}^{\mathrm{pr}}\left(A, \hat{\mathbb{Z}}_{l}\right)$ for $K_{n}^{\mathrm{pr}}\left(\mathcal{M}(A), \hat{\mathbb{Z}}_{l}\right)$ and $G_{n}\left(A, \hat{\mathbb{Z}}_{l}\right)$ for $K_{n}\left(\mathcal{M}(A), \hat{\mathbb{Z}}_{l}\right)$.

(iii) For $\mathcal{C}=\mathcal{P}(Y)$ as in Example $1\left(\right.$ iii) we shall write $K_{n}^{\mathrm{pr}}\left(Y, \hat{\mathbb{Z}}_{l}\right)$ for $K_{n}^{\mathrm{pr}}\left(\mathcal{P}(Y), \hat{\mathbb{Z}}_{l}\right)$ and $K_{n}\left(Y, \hat{\mathbb{Z}}_{l}\right)$ for $K_{n}\left(\mathcal{P}(Y), \hat{\mathbb{Z}}_{l}\right)$.

(iv) For $\mathcal{C}=\mathcal{M}(Y)$ as in Example $1\left(\right.$ iv), we shall write $G_{n}^{\mathrm{pr}}\left(Y, \hat{\mathbb{Z}}_{l}\right)$ for $K_{n}^{\mathrm{pr}}\left(Y, \hat{\mathbb{Z}}_{l}\right)$ and $G_{n}\left(Y, \hat{\mathbb{Z}}_{l}\right)=K_{n}\left(\mathcal{M}(Y), \hat{\mathbb{Z}}_{l}\right)$.

Remark 2 From the results obtained earlier by this author for general exact categories, (see [15, Chapter 8] or [13]) we can already deduce the following for $\mathcal{P}\left(\Lambda_{\alpha}[T]\right)$ and $\mathcal{M}\left(\Lambda_{\alpha}[T]\right)$.

(i) From [15, Lemma 8.2.1], we have the following exact sequences for $n \geq 1$.

(a) $0 \longrightarrow{\underset{s}{\lim }}^{1} K_{n+1}\left(\Lambda_{\alpha}[T], \mathbb{Z} / l^{s}\right) \longrightarrow K_{n}^{\mathrm{pr}}\left(\Lambda_{\alpha}[T], \hat{\mathbb{Z}}_{l}\right) \longrightarrow K_{n}\left(\Lambda_{\alpha}[T], \hat{\mathbb{Z}}_{l}\right) \longrightarrow 0$

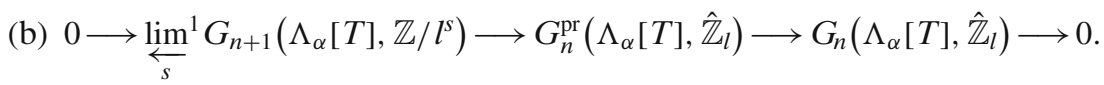

(ii) From [15, Theorem 8.2.2] we have for all $n \geq 2$,

(a) ${\underset{\lim }{\leftarrow}}_{s} K_{n}^{\mathrm{pr}}\left(\Lambda_{\alpha}[T], \hat{\mathbb{Z}}_{l}\right)\left[l^{s}\right]=0 ; \quad \overleftarrow{s}_{\lim ^{1}} K_{n+1}\left(\Lambda_{\alpha}[T], \mathbb{Z} / l^{s}\right)=\operatorname{div} K_{n}^{\mathrm{pr}}\left(\Lambda_{\alpha}[T], \hat{\mathbb{Z}}_{l}\right) ;$

(b) $\underset{s}{\lim } G_{n}^{\mathrm{pr}}\left(\Lambda_{\alpha}[T], \hat{\mathbb{Z}}_{l}\right)\left[l^{s}\right]=0 ; \quad{\underset{s}{\lim ^{1}}}^{1} G_{n+1}\left(\Lambda_{\alpha}[T], \mathbb{Z} / l^{s}\right)=\operatorname{div} G_{n}^{\mathrm{pr}}\left(\Lambda_{\alpha}[T], \hat{\mathbb{Z}}_{l}\right)$.

(iii) From [15, Lemma 8.2.2] or [13], we have

$$
\begin{aligned}
& \text { (a) } \lim _{s} K_{n}^{\mathrm{pr}}\left(\Lambda_{\alpha}[T], \hat{\mathbb{Z}}_{l}\right) / l^{s} \simeq K_{n}\left(\Lambda_{\alpha}[T], \hat{\mathbb{Z}}_{l}\right) ; \\
& \text { (b) }{\underset{\leftarrow}{s}}_{\lim } G_{n}^{\mathrm{pr}}\left(\Lambda_{\alpha}[T], \hat{\mathbb{Z}}_{l}\right) / l^{s} \simeq G_{n}\left(\Lambda_{\alpha}[T], \hat{\mathbb{Z}}_{l}\right)
\end{aligned}
$$

\subsection{Some Computations}

3.3.1 The aim of this subsection is to prove Theorem 8 below. Before stating the result, we first explain the construction of map $\varphi$ in Theorem 8(c) below.

Note that for any exact category $\mathcal{C}$, the natural map $M_{l^{\infty}}^{n+1} \rightarrow S^{n+1}$ induces a map

$$
\begin{gathered}
{\left[S^{n+1}, B Q \mathcal{C}\right] \stackrel{\varphi}{\longrightarrow}\left[M_{l \infty}^{n+1}, B Q \mathcal{C}\right], \quad \text { i.e., }} \\
K_{n}(\mathcal{C}) \stackrel{\varphi}{\longrightarrow} K_{n}^{\mathrm{pr}}\left(\mathcal{C}, \hat{\mathbb{Z}}_{l}\right)
\end{gathered}
$$

So when $\mathcal{C}=\mathcal{M}\left(\Lambda_{\alpha}[T]\right)$ we have a map

$$
\varphi: G_{n}\left(\Lambda_{\alpha}[T]\right) \longrightarrow G_{n}^{\mathrm{pr}}\left(\Lambda_{\alpha}[T], \hat{\mathbb{Z}}_{l}\right) .
$$


Theorem 8 Let $R$ be the ring of integers in a number field $F, \Lambda$ any $R$-order in a semi-simple F-algebra $\Sigma, \alpha: \Lambda \rightarrow \Lambda$ an R-automorphism of $\Lambda, \Lambda_{\alpha}[T]$ the $\alpha$-twisted Laurent series ring over $\Lambda$. Then, for all $n \geq 2$ :

(a) $\operatorname{div} G_{n}^{\mathrm{pr}}\left(\Lambda_{\alpha}[T], \hat{\mathbb{Z}}_{l}\right)=0$.

(b) $G_{n}^{\mathrm{pr}}\left(\Lambda_{\alpha}[T], \hat{\mathbb{Z}}_{l}\right) \simeq G_{n}\left(\Lambda_{\alpha}[T], \hat{\mathbb{Z}}_{l}\right)$ is an l-complete profinite Abelian group.

(c) The map $G_{n}\left(\Lambda_{\alpha}[T]\right) \longrightarrow G_{n}^{\mathrm{pr}}\left(\Lambda_{\alpha}[T], \hat{\mathbb{Z}}_{l}\right)$ is injective with uniquely l-divisible cokernel.

Proof (a) From Remark 2(ii)(b), we have

$$
\overleftarrow{\lim }_{s}^{1} G_{n+1}\left(\Lambda_{\alpha}[T], \mathbb{Z} / l^{s}\right)=\operatorname{div} G_{n}^{\mathrm{pr}}\left(\Lambda_{\alpha}[T], \hat{\mathbb{Z}}_{l}\right),
$$

for all $n \geq 2$. Now, by Theorem 1 (a) $G_{n}\left(\Lambda_{\alpha}[T]\right)$ is finitely generated for all $n \geq 1$. Hence $G_{n}\left(\Lambda_{\alpha}[T], \mathbb{Z} / l^{S}\right)$ is finite for all $n \geq 1$. In particular, $G_{n+1}\left(\Lambda_{\alpha}[T], \mathbb{Z} / l^{S}\right)$ is finite for all $n \geq 2$ and so $\lim _{s} G_{n+1}\left(\Lambda_{\alpha}[T], \mathbb{Z} / l^{s}\right)=0$ for all $n \geq 2$. Hence from Eq. 8, $\operatorname{div} G_{n}^{\mathrm{pr}}\left(\Lambda_{\alpha}[T], \hat{\mathbb{Z}}_{l}\right)=0$ for all $n \geq 2$.

(b) We saw in (a) above that $G_{n}\left(\Lambda_{\alpha}[T], \mathbb{Z} / l^{s}\right)$ is a finite group for all $n \geq 1$. Hence in the exact sequence

$$
0 \longrightarrow{\underset{s}{\lim }}^{1} G_{n+1}\left(\Lambda_{\alpha}[T], \mathbb{Z} / l^{s}\right) \longrightarrow G_{n}^{\mathrm{pr}}\left(\Lambda_{\alpha}[T], \hat{\mathbb{Z}}_{l}\right) \longrightarrow G_{n}\left(\Lambda_{\alpha}[T], \hat{\mathbb{Z}}_{l}\right) \longrightarrow 0
$$

we have $\lim ^{1} G_{n+1}\left(\Lambda_{\alpha}[T], \mathbb{Z} / l^{s}\right)=0$. Hence,

$$
G_{n}^{\mathrm{pr}}\left(\Lambda_{\alpha}[T], \hat{\mathbb{Z}}_{l}\right) \simeq G_{n}\left(\Lambda_{\alpha}[T], \hat{\mathbb{Z}}_{l}\right) .
$$

Now, by Remark 2(ii)(b),

$$
G_{n}^{\mathrm{pr}}\left(\Lambda_{\alpha}[T], \hat{\mathbb{Z}}_{l}\right) / l^{s} \simeq G_{n}\left(\Lambda_{\alpha}[T], \hat{\mathbb{Z}}_{l}\right) .
$$

So, from Eqs. 9 and $10 G_{n}^{\mathrm{pr}}\left(\Lambda_{\alpha}[T], \hat{\mathbb{Z}}_{l}\right) / l^{s} \simeq G_{n}^{\mathrm{pr}}\left(\Lambda_{\alpha}[T], \hat{\mathbb{Z}}_{l}\right)$ i.e. $G_{n}^{\mathrm{pr}}\left(\Lambda_{\alpha}[T], \hat{\mathbb{Z}}_{l}\right) \simeq$ $G_{n}\left(\Lambda_{\alpha}[T], \hat{\mathbb{Z}}_{l}\right)$ is $l$-complete. It is profinite since $G_{n}\left(\Lambda_{\alpha}[T], \hat{\mathbb{Z}}_{l}\right)=\lim _{\leftarrow} G_{n}\left(\Lambda_{\alpha}[T]\right.$, $\left.\mathbb{Z} / l^{S}\right)$ where each $G_{n}\left(\Lambda_{\alpha}[T], \mathbb{Z} / l^{S}\right)$ is a finite group.

(c) Since for all $n \geq 1, G_{n}\left(\Lambda_{\alpha}[T]\right)$ is a finitely generated Abelian group (see 2.1.1(a)), it follows that $G_{n}\left(\Lambda_{\alpha}[T]\right)$ is a finite group for each $n$. Hence $G_{n}\left(\Lambda_{\alpha}[T]\right)_{l}$ has no non-trivial divisible subgroups. Hence by [15, Corollary 8.2.1] or [13], kernel and cokernel of $\varphi$ are uniquely $l$-divisible. But $G_{n}\left(\Lambda_{\alpha}[T]\right)$ is finitely generated and so, $\operatorname{ker} \phi=\operatorname{div} \operatorname{ker} \phi=0$, as subgroups of $G_{n}\left(\Lambda_{\alpha}[T]\right)$.

\section{4. $K_{-1}(\Lambda), K_{-1}\left(\Lambda_{\alpha}[T]\right), \Lambda$ Arbitrary Orders}

\subsection{Finite Generation of $K_{-1}(\Lambda), K_{-1}\left(\Lambda_{\alpha}[T]\right)$.}

Let $R$ be the ring of integers in a number field $F, \Lambda$ any $R$-order in a semi-simple $F$-algebra $\Sigma, \alpha: \Lambda \rightarrow \Lambda$ and $R$-automorphism of $\Lambda, \Lambda_{\alpha}[T]$, the $\alpha$-twisted Laurent polynomial ring over $\Lambda$. We prove in this section that $K_{-1}(\Lambda)$ and $K_{-1}\left(\Lambda_{\alpha}[T]\right)$ are finitely generated Abelian groups for arbitrary $R$-orders $\Lambda$ in semi-simple $F$ algebras. Note that the proof in [9] by Farrell/Jones is for $\Lambda=\mathbb{Z} G, G$ a finite group. 
Also D. Carter shows in [4] that $K_{-1}(R G)$ is finitely generated and here we show that this result also holds more generally for arbitrary orders.

Finally we prove also that $N K_{-1}(\Lambda, \alpha)=0$ and so, $K_{-1}\left(\Lambda_{\alpha}[t]\right) \simeq K_{-1}(\Lambda)$.

Theorem 9 Let $F$ be an algebraic number field with ring of integers $R, \Lambda$ any $R$ order in a semi-simple F-algebra $\Sigma, \alpha: \Lambda \rightarrow \Lambda$ an R-automorphism of $\Lambda, \Lambda_{\alpha}[T]$ the $\alpha$-twisted Laurent series ring over $\Lambda$. Then

(a) $K_{-1}(\Lambda)$ is a finitely generated Abelian group.

(b) $K_{-1}\left(\Lambda_{\alpha}[T]\right)$ is a finitely generated Abelian group.

(c) $K_{-1}(\Lambda) \simeq K_{-1}\left(\Lambda_{\alpha}[t]\right)$.

Proof (a) Let $\Gamma$ be a maximal $R$-order containing $\Lambda$. Then, there exists a positive integer $s$ such that $s \Gamma \subset \Lambda$. Then $\underline{q}=s \Gamma$ is an ideal of $\Lambda$ and $\Gamma$. Put $B=\Lambda / q$, $B^{\prime}=\Gamma / \underline{q}$. Then we have a cartesian square

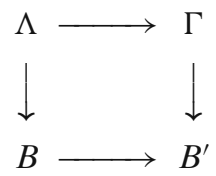

and hence a Mayer-Vietoris sequence

$$
\begin{aligned}
\cdots \rightarrow K_{1}\left(B^{\prime}\right) & \longrightarrow K_{0}(\Lambda) \longrightarrow K_{0}(\Gamma) \oplus K_{0}(B) \longrightarrow K_{0}\left(B^{\prime}\right) \\
& \longrightarrow K_{-1}(\Lambda) \longrightarrow K_{-1}(\Gamma) \oplus K_{-1}(B) \rightarrow \cdots
\end{aligned}
$$

Now by [1, Prop. 10.1, p. 685], $K_{-i}(A)=0$ for $i \geq 1$ and any quasi-regular ring $A$. Note that $B, B^{\prime}$ are finite rings and hence quasi-regular. Also $\Gamma$ is quasi-regular. Hence for $A=B, B^{\prime}$ or $\Gamma, K_{-i}(A)=0$ for $i \geq 1$. So the sequence Eq. 11 becomes

$$
\cdots \longrightarrow K_{0}(\Lambda) \longrightarrow K_{0}(\Gamma) \oplus K_{0}(B) \longrightarrow K_{0}\left(B^{\prime}\right) \longrightarrow K_{-1}(\Lambda) \longrightarrow 0
$$

To show that $K_{-1}(\Lambda)$ is finitely generated it suffices from Eq. 12 to show that $K_{0}\left(B^{\prime}\right)$ is finitely generated. Now $B^{\prime}$ is a finite Artinian ring and so, by [1, p. 465], $K_{0}\left(B^{\prime}\right) \simeq K_{0}\left(B^{\prime} / J B^{\prime}\right)$ where $J B^{\prime}=$ radical of $B^{\prime}$. But $B^{\prime} / J B^{\prime}$ is a finite semi-simple ring and so, $K_{0}\left(B^{\prime}\right) \simeq K_{0}\left(B^{\prime} / J B^{\prime}\right)$ is a finite direct sum of $K_{0}$ of (finite) fields each of which is isomorphic to $\mathbb{Z}$. Hence $K_{0}\left(B^{\prime}\right)$ is a (free) Abelian group of finite rank and hence is finitely generated. Hence $K_{-1}(\Lambda)$ is finitely generated.

(b) Let $\Gamma$ be an $\alpha$-invariant order containing $\Lambda$ as in Corollary 1. Let $s$ be a positive integer such that $s \Gamma \subset \Lambda$ and put $q=s \Gamma, B=\Lambda / q, B^{\prime}=\Gamma / q$. Then we have cartesian squares

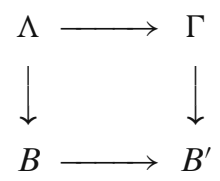


and

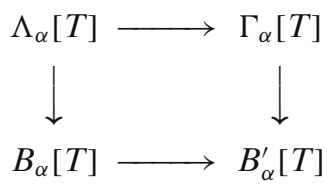

and hence a Mayer-Vietoris sequence

$$
\begin{gathered}
\cdots \longrightarrow K_{0}\left(\Lambda_{\alpha}[T]\right) \longrightarrow K_{0}\left(\Gamma_{\alpha}[T]\right) \oplus K_{0}\left(B_{\alpha}[T]\right) \\
\longrightarrow K_{0}\left(B_{\alpha}^{\prime}[T]\right) \longrightarrow K_{-1}\left(\Lambda_{\alpha}[T]\right) \longrightarrow 0 .
\end{gathered}
$$

where $\Gamma_{\alpha}[T], B_{\alpha}[T]$ and $B_{\alpha}^{\prime}[T]$ are quasi-regular (see [9]). If $A=\Gamma_{\alpha}[T], B_{\alpha}[T]$ or $B_{\alpha}^{\prime}[T]$ and $T^{n}$ is the free Abelian group of rank $n$. Then by [1, Prop. 10.1], $K_{-i}(A)=0$ for $i \geq 1$.

Also, by Serre's theorem $K_{0}(A) \rightarrow K_{0}\left(A\left[T^{n}\right]\right)$ is an epimorphism (see [7]). Since $K_{-n}(A)$ is a direct summand of the cokernel of $K_{0}(A) \rightarrow K_{0}\left(A\left[T^{n}\right]\right)$ we have $K_{-n}(A)=0$ for $n \geq 1$. So from the exact sequence Eq. 11 , we have $K_{-n}\left(\Lambda_{\alpha}[T]\right)=0$ for $n \geq 2$ and $K_{0}\left(\bar{B}_{\alpha}^{\prime}[T]\right) \longrightarrow K_{-1}\left(\Lambda_{\alpha}[T]\right)$ is an epimorphism.

By mapping the Mayer-Vietoris sequence associated with the cartesian square Eq. 11 to the Mayer-Vietoris sequence associated with square Eq. 12, we have a commutative square

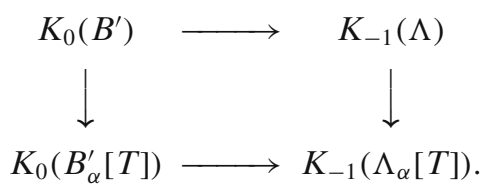

To prove that $K_{-1}(\Lambda) \longrightarrow K_{-1}\left(\Lambda_{\alpha}[T]\right)$ is an epimorphism, it suffices to prove that $K_{0}\left(B^{\prime}\right) \longrightarrow K_{0}\left(B_{\alpha}^{\prime}[T]\right)$ is an epimorphism in the commutative diagram

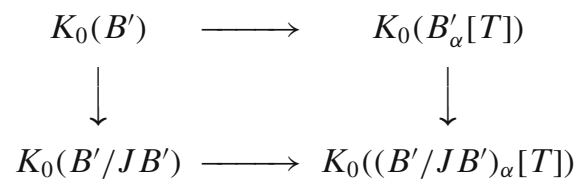

where the vertical maps are isomorphisms. Also by [7, Theorem 27], the map $K_{0}\left(B^{\prime} /\right.$ $\left.J B^{\prime}\right) \longrightarrow K_{0}\left(\left(B^{\prime} / J B^{\prime}\right)_{\alpha}[T]\right)$ is an epimorphism. Hence $K_{0}\left(B^{\prime}\right) \longrightarrow K_{0}\left(B_{\alpha}^{\prime}[T]\right)$ is an epimorphism. So $K_{-1}(\Lambda) \longrightarrow K_{-1}\left(\Lambda_{\alpha}[T]\right)$ is an epimorphism. Since by (a), $K_{-1}(\Lambda)$ is finitely generated, then $K_{-1}\left(\Lambda_{\alpha}[T]\right)$ is also finitely generated.

(c) By definition, $K_{-1}\left(\Lambda_{\alpha}[t]\right) \simeq K_{-1}(\Lambda) \oplus N K_{-1}(\Lambda, \alpha)$. So it suffices to show that $N K_{-1}(\Lambda, \alpha)=0$.

Let $\Lambda, \Gamma, B=\Lambda / q, B^{\prime}=\Gamma / q$ be as in the proof of (a) (b). Then we have two cartesian squares

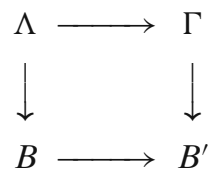


and

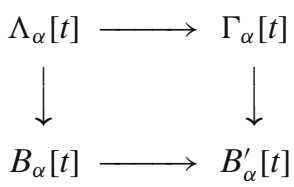

where $\Gamma_{\alpha}[t], B_{\alpha}[t]$ and $B_{\alpha}^{\prime}[t]$ are quasi-regular as well as $\Gamma, B, B^{\prime}$. Hence we have Mayer-Vietoris sequences

$$
\cdots \rightarrow K_{0}\left(\Lambda_{\alpha}[t]\right) \longrightarrow K_{0}\left(\Gamma_{\alpha}[t]\right) \oplus K_{0}\left(B_{\alpha}[t]\right) \longrightarrow K_{0}\left(B_{\alpha}^{\prime}[t]\right) \longrightarrow K_{-1}\left(\Lambda_{\alpha}[t]\right) \rightarrow \ldots
$$

and

$$
\cdots \rightarrow K_{0}(\Lambda) \longrightarrow K_{0}(\Gamma) \longrightarrow K_{0}(B) \longrightarrow K_{0}\left(B^{\prime}\right) \longrightarrow K_{-1}(\Lambda) \rightarrow \cdots
$$

where for $A=\Gamma, B, B^{\prime}, \Gamma_{\alpha}[t], B_{\alpha}[t], B_{\alpha}^{\prime}[t], K_{-i}(A)=0$ for $i \geq 1$ (see [1, Prop. 10.1]). By mapping Eqs. 19 to 20 and taking kernels, we have that

$$
N K_{-1}(\Lambda, \alpha)=\operatorname{coker}\left(N K_{0}(\Gamma, \alpha) \oplus N K_{0}(B, \alpha) \longrightarrow N K_{0}\left(B^{\prime}, \alpha\right)\right) .
$$

So it suffices to show that $N K_{0}\left(B^{\prime}, \alpha\right)=0$. Since $B^{\prime}, B_{\alpha}^{\prime}[t]$ are quasi-regular, the result follows from [6, Lemma 2.4]. So $N K_{-1}(\Lambda, \alpha)=0$ and hence $K_{-1}(\Lambda[t]) \simeq K_{-1}(\Lambda)$.

Corollary 2 let $R$ be the ring of integers in a number field $F, V=G \rtimes_{\alpha} T$ a virtually infinite cyclic group where $G$ is a finite group and the action of the infinite cyclic group $T$ on $G$ is given by $\alpha(g)=\operatorname{tgt}^{-1}$ for all $g \in G$. Then $K_{-1}(R V)$ is a finitely generated Abelian group.

Corollary 3 Let $\alpha$ be an automorphism of a finite group $G, R$ the ring of integers in a number field $F$. Denote the induced automorphism on $R G$ also by $\alpha$. Then $K_{-1}(R G) \simeq K_{-1}\left((R G)_{\alpha}[t]\right)$ is a finitely generated Abelian group.

Acknowledgements Part of the work reported in this article was done while I was visiting University of Bielefeld, Germany and IHES, Paris. It was concluded and written up while I was visiting the Max-Planck-Institut für Mathematik, Bonn, Germany. I like to thank the three institutions for hospitality and financial support.

\section{References}

1. Bass, H.: Algebraic $K$-theory. W.A. Benjamin, Menlo Park (1968)

2. Borel, A.: Stable real cohomology of arithmetic groups. Ann. Sci. École Norm. Sup. 7(4), 235-272 (1984)

3. Browder, W.: Algebraic $K$-theory with Coefficients $\mathbb{Z} / p$. Lecture Notes in Mathematics, vol. 657, pp. 40-84. Springer, Berlin (1978)

4. Carter, D.W.: Localization in lower algebraic $K$-theory. Comm. Algebra 8(7), 603-622 (1980)

5. Charney, R.: A Note on Excision in $K$-theory. Lecture Notes in Mathematics, vol. 1046, pp. 47-48. Springer, Berlin (1984)

6. Conolly, F., Prassidis, S.: On the exponents of $N K$ groups of virtually infinite cyclic groups. Car. Math. Bull. 45(2), 180-195 (2002)

7. Farrell, F.T., Hsiang, W.C.: A formula for $K_{1}(R \alpha[T])$. Applications of Categorical Algebra. Proceedings of Symposia in Pure Mathematics, vol. 17, pp. 192-218. American Mathematical Society, Providence (1970) 
8. Farrell, F.T., Jones, L.E.: Isomorphisms conjectures in algebraic $K$-theory. J. Amer. Math. Soc. 6, 249-297 (1993)

9. Farrell, F.T., Jones, L.E.: The lower algebraic $K$-theory of virtually infinite cyclic groups. K-Theory 9, 13-30 (1995)

10. Kuku, A.O. $K$-theory of grouprings of finite groups over maximal orders in division algebras. J. Algebra 91(1), 18-31 (1985)

11. Kuku, A.O.: $K_{n}, S K_{n}$ of integral group rings and orders. Contemp. Math. AMS 55, 333-338 (1986)

12. Kuku, A.O.: Ranks of $K_{n}$ and $G_{n}$ of orders and grouprings of finite groups over integers in number fields. J. Pure Appl. Algebra 138, 39-44 (1999)

13. Kuku, A.O.: Profinite and continuous higher $K$-theory of exact categories, orders and group rings. K-theory 22, 367-392 (2001)

14. Kuku, A.O.: Finiteness of higher $K$-groups of orders and group rings. K-Theory 36, 51-58 (2005)

15. Kuku, A.O.: Representation Theory and Higher Algebraic K-theory. Chapman and Hall, London (2007)

16. Kuku, A.O., Tang, G.: Higher $K$-theory of grouprings of virtually infinite cyclic groups. Math. Ann. 323, 711-725 (2003)

17. Neisendorfer, J.: Primary homotopy theory. Mem. Amer. Math. Soc. 232, (1980)

18. Reiner, I.: Maximal Orders. Academic Press, London (1975)

19. Weibel, C.: Mayer-Vietoris sequences and mod- $p$ - $K$-Theory. Lecture Notes in Mathematics, vol. 966, pp. 390-407. Springer, Berlin (1982) 